03

\title{
Исследование внутренней структуры микрорезонаторов методом оптической томографии
}

\author{
(ㄷ Г.Г. Левин ${ }^{1}$, В.Л. Минаев ${ }^{1, \text { ॠ К.Н. Миньков }}{ }^{1,2}$, М.М. Ермаков ${ }^{1}$, А.А. Самойленко ${ }^{1}$ \\ ${ }^{1}$ Всероссийский научно-исследовательский институт оптико-фризических измерений, \\ 119361 Москва, Россия \\ ${ }^{2}$ Московский институт электроники и математики им. А.Н. Тихонова, \\ Национальный исследовательский университет высшей школы экономики, \\ 101000 Москва, Россия \\ Te-mail: minaev@vniiofi.ru \\ Поступила в редакцию 04.06.2018 г. \\ В окончательной редакции 25.10.2018 г. \\ Принята к публикации 06.11.2018 г.
}

Разработан микроскоп, который позволяет исследовать внутренние неоднородности показателя преломления оптических диэлектрических микрорезонаторов методом оптической томографии. Экспериментально исследовано влияние внутренних неоднородностей показателя преломления оптических диэлектрических микрорезонаторов, изготовленных методом термообработки, на их добротность.

DOI: $10.21883 /$ OS.2019.03.47371.148-18

\section{Введение}

Развитие новых технологий создания изделий с заранее заданными свойствами требует разработки новых систем диагностики и измерений различных характеристик этих изделий, в том числе и их внутренней структуры. Например, при изготовлении объектов с использованием аддитивных технологий, которые в последнее десятилетие очень активно развиваются, требуется не только измерить форму и структуру поверхности, но также и однородность материала внутри изделия. В настоящее время все больше появляется методов изготовления оптических деталей с использованием аддитивных технологий, что позволяет создавать элементы произвольной формы с переменным показателем преломления внутри [1]. Аналогичные задачи возникают при изготовлении объектов из растворов и расплавов различных веществ. В частности, при создании оптоволокна с переменным профилем показателя преломления необходимо контролировать его распределение как в готовых изделиях, так и в заготовках [2]. При этом важным является получение количественных данных о локальных значениях показателя преломления внутри объекта и о пространственном распределении его неоднородностей. Поэтому разработка метода измерений указанных характеристик является очень важной задачей. Во всех случаях, когда в качестве исходного сырья используется прозрачный материал, для исследования распределения показателя преломления и определения его количественных значений наиболее перспективны методы оптической томографии.

В настоящей работе рассмотрена возможность исследования внутренней структуры оптически прозрачных объектов на примере оптического микрорезонатоpa [3]. Оптические диэлектрические микрорезонаторы с модами типа „шепчущей галереи“ сочетают в себе уникальные характеристики: высокую добротность и стабильность частот мод, высокую концентрацию поля в малом объеме внутри резонатора и у его поверхности, возможность создания микрорезонаторов для широкого диапазона длин волн с применением диэлектрических материалов, стойких к воздействию окружающей среды. Это дает возможность использовать микрорезонаторы в качестве эффективных преобразователей малых изменений параметров окружающей среды в изменение частоты, измеряемое с высокой точностью. Особую роль такие изделия могут играть в составе сенсоров, предназначенных для обнаружения наноразмерных объектов: наночастиц и биологических субстанций [4].

Одним из ключевых параметров микрорезонатора является добротность моды шепчущей галереи. В значительной степени добротность определяется рассеянием оптического излучения на различных дефектах, с которыми может взаимодействовать излучение моды. Рассеяние оптического излучения происходит на любых локальных неоднородностях показателя преломления. При этом важно определять не только расположение его флуктуаций, но и их абсолютное значение. Особенно важным контроль локальных неоднородностей показателя преломления является для микрорезонаторов, изготовленных методом термообработки, который подразумевает расплавление материала. Остаточные загрязнения на поверхности заготовки могут вплавиться в микрорезонатор и создать локальные вариации показателя преломления. При этом необходимо провести измерения локальных микроскопических неоднородностей показателя преломления по всему объему микрорезонатора. Такие неоднородности невозможно зарегистрировать стандартными методами оптической или зондовой микроскопии, поэтому нами был выбран метод оптической томографии. 


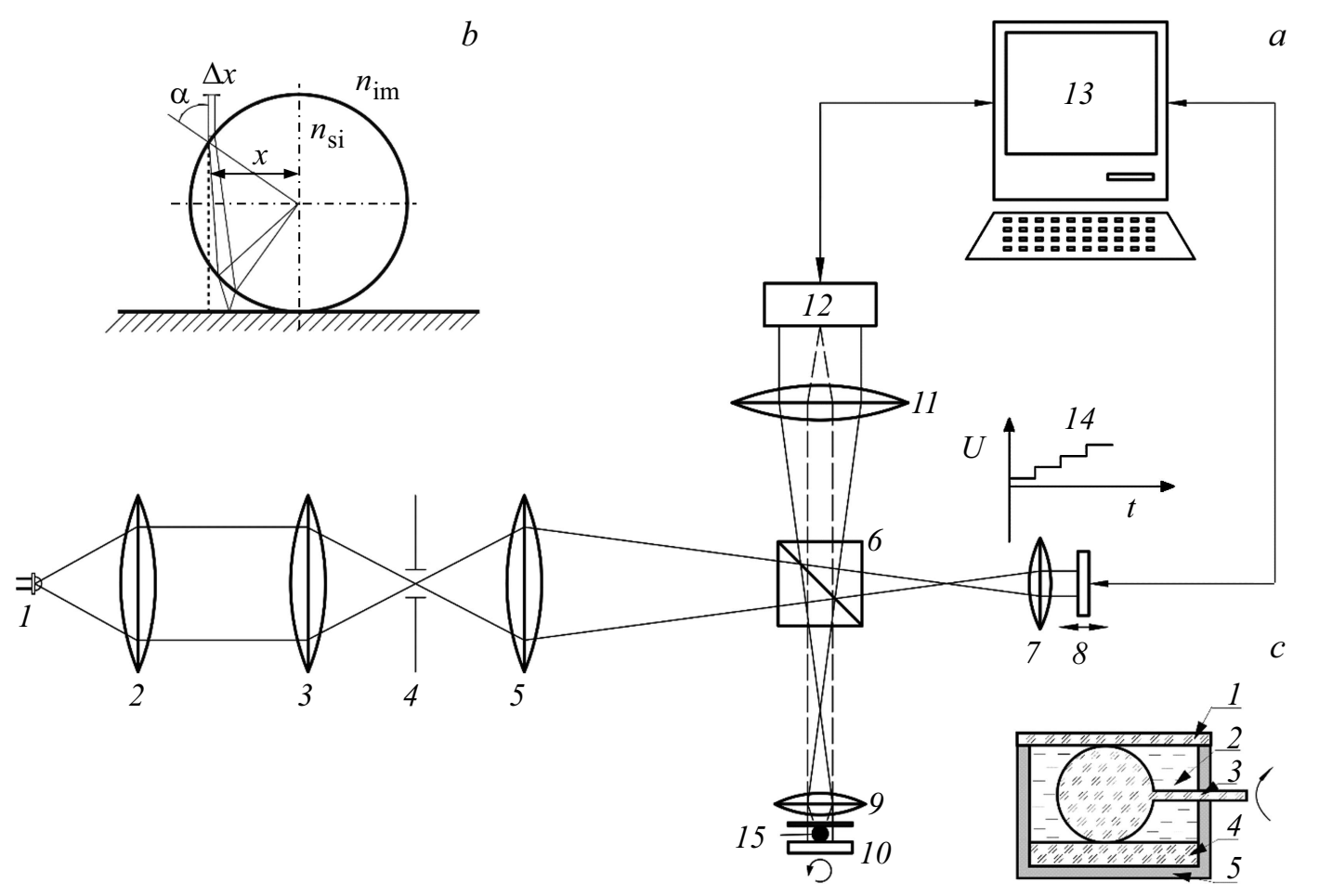

Рис. 1. а) Оптическая схема микроскопа: 1 - источник; 2,3,5 - линзы; 4 - диафрагма; 6 - светоделитель; 7,9 микрообъективы; 8 - опорное зеркало, 10 - зеркало; 11 - проекционная линза; 12 - матрицы видеокамеры; 13 персональный компьютер; 14 - график напряжения, подаваемого на пьезоэлемент опорного зеркала; 15 - микрорезонатор. b) Схема прохождения луча через микрорезонатор: $\alpha-$ угол падения луча; $\Delta x-$ поперечное смещение луча; $x-$ прицельный параметр. c) Кювета с микрорезонатором: 1 - покровное стекло; 2 - глицерин; 3 - микрорезонатор; 4 - зеркало; 5 - кювета.

Для реконструкции пространственного распределения показателя преломления внутри прозрачного объекта необходимо а) разработать оптическую систему, позволяющую получать число проекций, достаточное для восстановления функции, которая описывает это распределение в зависимости от числа неоднородностей внутри объекта и их градиента, б) разработать схему интерференционного прибора, позволяющего восстанавливать из интерферограмм распределение фазы, которое описывает проекционные данные с точностью, необходимой для решения обратной задачи томографии. Для этого была выбрана схема автоматизированного интерферометра Линника (рис. 1,a), которая позволяет измерять фазу волнового фронта с погрешностью $0.1 \%$, что необходимо для решения обратной некорректной задачи реконструкции томограмм [5]. В данной схеме изображение источника излучения 1 (светодиод с центральной длиной волны $530 \mathrm{~nm}$ ) с помощью оптической системы 2 и 3 строится в плоскости апертурной диафрагмы 4, а затем с помощью конденсорной линзы 5 отображается в передние фокальные плоскости микрообъективов 7 и 9 после предварительного разделения на предметный и опорный пучки с помощью светоделителя 6 . Предметный пучок (вертикальный) падает на исследуемый микрорезонатор 15 (показан условно). Микрорезонатор представляет собой кварцевую сферу размером 200-500 $\mu \mathrm{m}$ на стек- лянной „ножке“. Для компенсации рефракции на границе микрорезонатор помещается в кювету с иммерсионной жидкостью, которая имеет зеркальную нижнюю грань 4, и накрывался покровным стеклом 1 (рис. 1,c). Микрорезонатор имеет возможность вращения вокруг своей оси. Опорный пучок (горизонтальный) падает на опорное зеркало 8, которое соединено с пьезоэлементом (не указан). Оба пучка (предметный и опорный), отразившись от зеркал 10 и 8 , проходят через светоделитель 6 , проекционную линзу 11 и строят изображение предмета и опорного зеркала в плоскости фотодетектора матрицы видеокамеры 12. Видеокамера и пьезоэлемент опорного зеркала управляются от ПЭВМ 13.

Для получения фазовых изображений (проекций) использовался метод фазовых шагов [6]. Для этого интерференционные изображения при различных фазовых сдвигах зеркала 8 , реализуемых путем подачи дискретных значений напряжения на пьезоэлемент 14, записывались в ПЭВМ. Далее с помощью специального ПО „WinPhast“ происходила расшифровка записанных интерферограмм и восстановление фазового изображения.

\section{1. Расчет влияния рефракции}

При разработке оптического томографа, который позволил бы решить задачу реконструкции распределения 
показателя преломления внутри объекта, необходимо разработать такую схему зондирования, при которой полученные данные представляют собой проекции, описываемые преобразованием Радона. В интерферометрии условия, при которых можно пренебречь искривлением траектории зондирующего излучения и дифракционными эффектами, вызванными внутренними неоднородностями, подробно рассматривались ранее в [7] при оценке влияния рефракции на результаты реконструкции томограмм. В случае исследования твердых тел, какими являются изделия аддитивных технологий, оптоволокна, микрорезонаторы и другие прозрачные объекты, особое влияние оказывает преломление на границе объекта. Поэтому при измерении характеристик внутренней структуры таких объектов методами оптической томографии важную роль играет показатель преломления среды, в которую помещается объект. Как правило, в качестве такой среды выбирается иммерсия с показателем преломления, близким к показателю преломления исследуемого объекта. Однако практически невозможно подобрать иммерсию, полностью исключающую преломление света на границе объекта. Далее мы рассмотрим влияние преломления света на границе исследуемого объекта для данной схемы интерференционного микроскопа.

В микроскопе измеряется распределение фазы излучения, прошедшего через объект, которое связано с распределением показателя преломления следующим выражением:

$$
\phi(x, y)=\frac{4 \pi}{\lambda}\left(n_{\mathrm{im}} l-n_{\mathrm{im}}\left(1-L(x, y)+\int_{L(x, y)} n(x, y, z) d z\right)\right.
$$

где $4 \pi$ связано с двойным прохождением излучения через исследуемый объект (схема на отражение); $n_{i m}-$ показатель преломления иммерсии; $l-$ вертикальный размер кюветы с иммерсией, в которую помещен объект; $n(x, y, z)$ - распределение показателя преломления объекта; $L(x, y)$ - размер объекта вдоль оси $z$ (направление зондирования).

Если в данном выражении последнее слагаемое представляет собой преобразование Радона, то можно применять методы реконструкции томограмм, основанные на инверсном преобразовании Радона. Для этого необходимо, чтобы выполнялись два условия. Во-первых, поперечное смещение луча в плоскости $(X, Y)$ (вдоль которого происходит интегрирование показателя преломления) возникающее из-за преломления лучей на разделе сред объект-иммерсия, должно быть в пределах одного воксела реконструируемой томограммы. При нарушении данного условия траектория луча зондирования, вдоль которого интегрируется искомая функция, будет интерпретирована неверно, что приведет к возникновению артефактов в томограмме.

Второе условие связано с детерминированным изменением значения измеряемой фазы, вызванным преломлением света на границе объект-иммерсия и, как следствие, увеличением оптической длины пути внутри объекта. В том случае, если изменение фазы, вызванное отклонением траектории луча от направления зондирования, превышает погрешность ее измерения, то в реконструированной томограмме также могут возникнуть артефакты.

Выполнить указанные требования можно, выбрав такую иммерсию, которая компенсировала бы эти погрешности измерения фазы в проекционных данных. Нетрудно заметить, что подбор иммерсии зависит от показателя преломления исследуемого объекта и его формы. В нашем случае форма объекта близка к сферической, a его средний показатель преломления определяется сырьем, из которого он изготавливался, что позволило подобрать показатель преломления иммерсии методом математического моделирования.

В расчетах за объект принимался шарик радиусом $250 \mu \mathrm{m}$, изготовленный из $\mathrm{SiO}_{2}$ с показателем преломления $n_{\mathrm{SI}}=1.45778$, освещаемый вертикальным параллельным пучком света с длиной волны $\lambda=650 \mathrm{~nm}$. Шарик находился на зеркальной поверхности и был окружен иммерсией с показателем преломления $n_{\mathrm{im}}$. Поэтому расчеты проводились с учетом того, что луч два раза проходил через шарик (рис. $1, b)$. Разность показателей преломления иммерсии и шарика $\Delta n=n_{\mathrm{SI}}-n_{\mathrm{im}}$. Пунктирной линией на рис. $1, b$ отмечен ход луча, когда $\Delta n=0$, т.е. луч не преломляется, при этом его оптическая длина пути составляла $d_{0}$, сплошные линии - ход лучей при $\Delta n=\Delta n_{\max }$, при этом оптическая длина пути составляла $d_{1}$. Расстояние от луча до оси шарика, так называемый прицельный параметр, равно $x$.

Были найдены зависимости поперечного смещения $\Delta x$ и относительной погрешности фазового набега $\delta$, определяемой как

$$
\delta=\frac{\left(d_{1}-d_{0}\right)}{d_{0}} 100 \%,
$$

от прицельного параметра $x$ при определенном значении $\Delta n_{\max }$.

Расчеты показали, что при значении $\Delta n_{\max }=0.002$ смещение луча не превышает размера одного воксела, который составляет $2 \mu \mathrm{m}$ (при сетке томографической реконструкции $250 \times 250 \times 250)$, а относительная погрешность фазового набега не превышает 1\% (рис. 2). При этом погрешность фазового набега носит неслучайный характер. Она возрастает к краю объекта, что может приводить к возникновению круговых артефактов в реконструированной томограмме [5]. Смещение луча также возрастает к краю объекта, но при выбранных параметрах иммерсии не приведет к существенным артефактам.

\section{2. Экспериментальные результаты}

Для выяснения связи между добротностью и внутренней структурой были изготовлены экспериментальные образцы микрорезонаторов с различной внутренней 

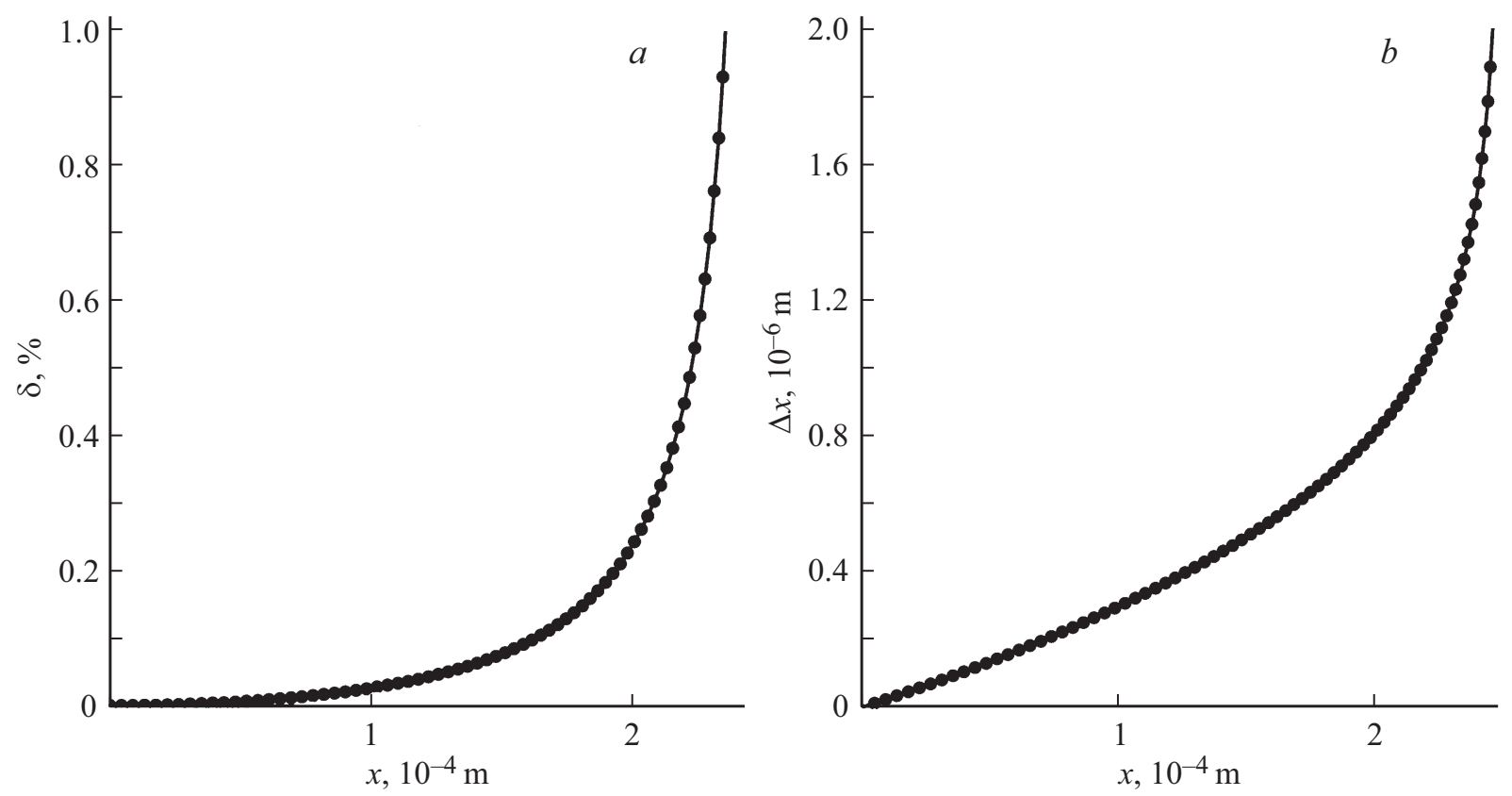

Рис. 2. Зависимости относительной погрешности фазового набега $\delta(a)$ и поперечного смещения луча $\Delta x(b)$ от прицельного параметра $x$ при $\Delta n_{\max }=0.002$.

структурой и проведены эксперименты по измерению их добротности и распределения показателя преломления в сечении.

Для изготовления микрорезонаторов было использовано оптическое волокно SM600 (Thorlabs Inc., США). Процесс изготовления микрорезонаторов термическим способом подробно описан в [3]. Как показали предварительные эксперименты, очень важно в момент изготовления микрорезонаторов контролировать параметры окружающей среды и количество наночастиц в окружающем воздухе. Для этого были использованы измеритель параметров микроклимата типа „Метеоскоп“ и аэрозольный счетчик частиц GEO- $\alpha$ 3886. Всего было изготовлено 5 различных микрорезонаторов. После изготовления микрорезонаторов была измерена их добротность. Процесс измерения добротности подробно описан в [8].

Следующим этапом было получение проекций. Для этого исследуемый микрорезонатор свободным концом вставлялся в медицинскую иглу с внутренним диаметром $300 \mu \mathrm{m}$ и в месте крепления заливался эпоксидным клеем. С помощью переходника игла закреплялась на механическом угловом позиционере марки 5РН51 (Standa, Литва).

Для того чтобы уменьшить относительный фазовый набег, микрорезонатор помещался в иммерсионную жидкость (глицерин) с показателем преломления $n_{\mathrm{im}}$, близким по значению к показателю преломления самого микрорезонатора. По оценкам разность показателей преломления $\Delta n$ составила не более 0.002 . Для получения проекций (фазовых изображений) использовался метод фазовых шагов [9]. Микроскоп был сфокусирован на зеркало, при этом наблюдались контрастные интерфе- ренционные полосы (рис. 3,a). Перед записью проекций было предварительно записано фазовое изображение без резонатора - фазовые аберрации микроскопа, которые затем вычитались из каждого измерения. Также по маске, исключающей область с резонатором, вычиталась наклонная плоскость (клин), обусловленная интерференцией в полосах конечной ширины.

C точки зрения томографии фазовое изображение $\Delta(x, y)$ является проекцией, которая несет информацию о внутренней структуре в интегральной форме при направлении зондирования вдоль оптической оси $z$ :

$$
\begin{aligned}
\Delta(p, y ; \alpha)= & \iint\left(n(x, y, z)-n_{\mathrm{im}}\right) \\
& \times \delta(p-x \cos (\alpha)-z \sin (\alpha)) d x d z,
\end{aligned}
$$

где $n(x, y, z)$ - трехмерное распределение показателя преломления микрорезонатора, $\alpha$ - угол поворота (вдоль оси $y$ ) при котором получена проекция.

Были получены 18 проекций резонатора в диапазоне углов от $0^{\circ}$ до $180^{\circ}$ с шагом $10^{\circ}$. Перед реконструкцией была проведена их предварительная обработка, позволившая вычислить и устранить продольный сдвиг проекций (вдоль оси $y$ ). Нулевой момент трехмерной функции $n(x, y, z)$, равный $M_{00}(y)$, вдоль оси $x$ не зависит от угла поворота $\alpha$ и равен нулевому момент от проекции вдоль оси $p$, равный $m_{0}(y ; \alpha)$. Поэтому для всех проекций их нулевые моменты $m_{0}(y ; \alpha)$ будут равны, но сдвинуты вдоль оси $y$. Эти сдвиги были найдены, и в расчеты внесена соответствующая корректировка. После этого были получены сечения в экваториальной области (имеющие одну и ту же координату $y$ ) (рис. $3, b$ ), которые были 

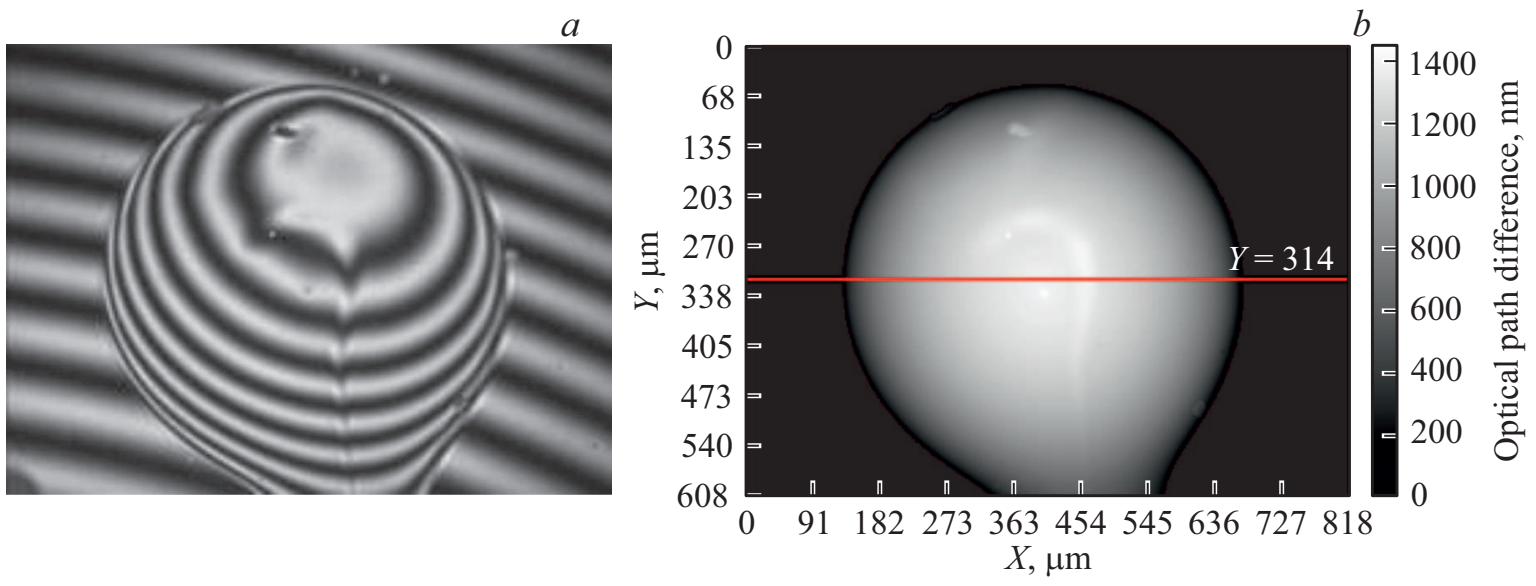

Рис. 3. Интерференционное $(a)$ и фазовое $(b)$ изображения микрорезонатора.
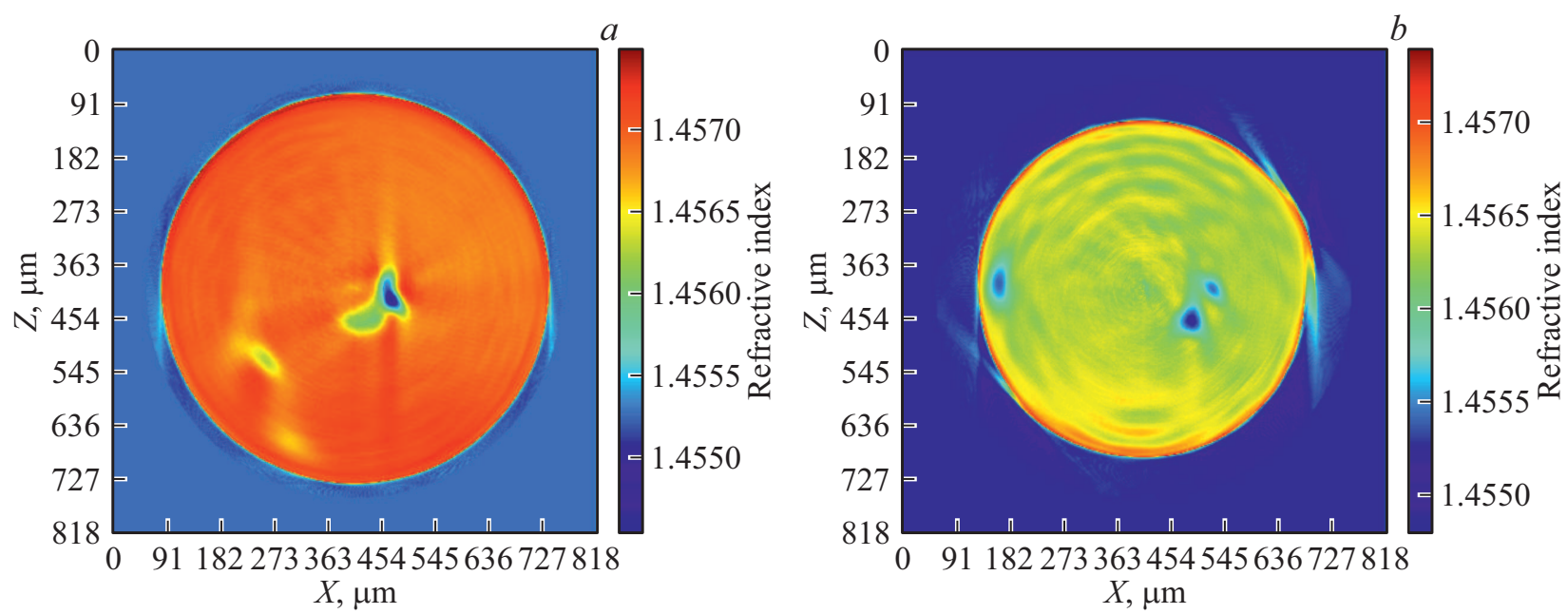

Рис. 4. Томограммы микрорезонаторов с различной добротностью: $3.5 \cdot 10^{9}(\mathrm{a}), 1.7 \cdot 10^{9}(\mathrm{~b})$.

Значение добротности и показателей преломления исследуемой партии микрорезонаторов

\begin{tabular}{c|c|c|c}
\hline № & Добротность & $\Delta n_{\text {spec }}$ & $\Delta n_{\text {meas }}$ \\
\hline 1 & $2.5 \cdot 10^{9}$ & $3.5 \cdot 10^{-3}$ & $2.0 \cdot 10^{-3}$ \\
2 & $1.1 \cdot 10^{9}$ & $3.5 \cdot 10^{-3}$ & $1.5 \cdot 10^{-3}$ \\
3 & $1.7 \cdot 10^{9}$ & $3.5 \cdot 10^{-3}$ & $1.0 \cdot 10^{-3}$ \\
4 & $2.8 \cdot 10^{9}$ & $3.5 \cdot 10^{-3}$ & $1.5 \cdot 10^{-3}$ \\
5 & $3.5 \cdot 10^{9}$ & $3.5 \cdot 10^{-3}$ & $2.5 \cdot 10^{-3}$
\end{tabular}

нормированы на нулевой момент и совмещены по их центрам тяжести.

Близость показателей преломления микрорезонатора и иммерсионной жидкости позволила использовать методы вычислительной томографии без учета рефракции. Реконструкция томограммы проводилась с помощью алгоритма SART [10], реализованного в составе пакета scikit-image 0.14 [11].
Результаты экспериментов приведены в таблице. В таблице указаны добротности микрорезонаторов, а также разность показателей преломления внутренней жилы и оболочки, полученной из спецификации для волокна SM600 $\Delta n_{\mathrm{spec}}$, а также измеренной томографическим методом $\Delta n_{\text {meas }}$.

На рис. 4 представлены примеры восстановленных томограмм микрорезонаторов с различной добротностью. На них видны неоднородности показателя преломления, связанные с наличием внутренней жилы. Из рис. 4, $a$ видно, что микрорезонатор с неоднородностью вблизи поверхности имеет более низкую добротность (рис. $4, b$ ).

\section{Заключение}

Результаты экспериментальных исследований позволяют делать следующие выводы. Первые томографические измерения показателя преломления внутри твердых тел позволяют определить изменения показателя преломления с погрешностью $10^{-3}-10^{-4}$. Это позволяет 
диагностировать отклонение от технологического процесса при изготовлении оптических деталей методами аддитивных технологий, которые получают широкое распространение в настоящее время.

Для построения оптического томографа необходимо выполнить два главных условия. Во-первых, использовать томографический интерферометр, позволяющий с высокой точностью измерять волновой фронт при достаточно большом числе ракурсов, не менее $10^{-15}$. Во-вторых, объект исследования должен быть помещен в кювету с иммерсионной жидкостью, что позволит компенсировать эффект рефракции на границе раздела двух сред. При этом показатель преломления иммерсии необходимо выбирать в зависимости от размера и формы объекта, при соблюдении условий минимизации отклонения луча и детерминированной погрешности фазы.

Работа выполнена с использованием оборудования ЦКП высокоточных измерительных технологий в области фотоники (ckp.vniiofi.ru), созданного на базе ФГУП „ВНИИОФИ“ и поддержке Министерства образования и науки Российской Федерации в рамках выполнения соглашения № 14.625.21.0041 от 26.09.2017г. (уникальный идентификатор прикладных научных исследований RFMEFI62517X0041). Часть исследований выполнена с привлечением оборудования ЦКП „Исследования наноструктурных, углеродных и сверхтвердых материалов“ ФГБНУ ТИСНУМ.

\section{Список литературы}

[1] Luo J. et al. // Proc. SPIE 9738. Laser 3D Manufacturing III. 2016. V. 9738. P. 97380Y-1-97380Y-9.

[2] Michael H. Frosz, Alessio Stefani, and Ole Bang Highly // Optics Expres, 2011. V. 19. N 1. P. 10471-10484.

[3] Городецкий М.Л. Оптические микрорезонаторы с гигантской добротностью. М.: Физматлит, 2011.

[4] Самойленко А.А., Левин Г.Г., Лясковский В.Л., Миньков К.Н., Иванов А.Д., Биленко И.А. // Опт. и спектр. 2017. B. 122. № 6. C. 1037-1039.

[5] Herman G. Image reconstruction from projections: the fundamentals of computerized tomography. N.Y.-London: Academic Press, 1980. V. XII. 316 p.

[6] Вишняков Г.Н., Левин Г.Г., Минаев В.Л. // Автометрия. 2017. T. 53. № 5. C. $131-138$.

[7] Левин Г.Г., Вишняков Г.Н. Оптическая томография. М.: Радио и связь, 1989. 224 с.

[8] Ружицкая Д.Д., Самойленко А.А., Иванов А.Д., Миньков К.Н. // Автометрия. 2018. Т. 54. № 1. С. 71-79.

[9] Vishnyakov G., Levin G., Minaev V., Nekrasov N. // Appl. Opt. 2015. V. 54. N 15. P. 4797-4804.

[10] Andersen A., Kak A. // Ultrasonic Imaging. 1984. N 6. P. 81-94.

[11] Van der Walt S., Schönberger J.L., Nunez-Iglesias J., Boulogne F., Warner J.D., Yager N., Gouillart E., Yu T. and the scikit-image contributors. scikit-image: Image processing in Python. PeerJ 2:e453 (2014). 